\title{
A multistatic circular synthethic aperture radar for small object detection
}

\begin{abstract}
This paper introduces a ground-based Multistatic Circular Synthetic Aperture Radar (MuCSAR) used to detect small objects on the ground. The received signals have been modelled and the system prototype has been developed. The proposed signal processing is also described. An experimental investigation for Foreign Object Detection application has been analysed.
\end{abstract}

Keyword: Multistatic Circular Synthetic Aperture Radar (MuCSAR); Small object detection; Signal 